\title{
Audit of cardiovascular risk assessment and lipid management by Canadian primary care physicians
}

\author{
Milan Gupta MD FRCPC FACC 1,2,3, Michelle Tsigoulis ${ }^{1}$, Mahesh Kajil MBBS1, \\ Subodh Verma MD PhD FRCSC FAHA ${ }^{3}$, Narendra Singh MD FRCPC FACC FAHA ${ }^{1,4}$
}

\begin{abstract}
M Gupta, M Tsigoulis, M Kajil, S Verma, N Singh. Audit of cardiovascular risk assessment and lipid management by Canadian primary care physicians. Curr Res Cardiol 2016;3(2):33-37.
\end{abstract}

BACKGROUND: Assessment of cardiovascular risk is an essential component of preventive cardiology. Despite guideline recommendations, risk assessment remains highly variable. Further efforts to understand the knowledge and action gaps for risk assessment and lipid management in contemporary primary care are warranted.

METHODS: A retrospective chart audit of 105 physicians participating in an observational registry of healthy middle-age Canadians free of known cardiovascular disease (CVD), diabetes or treated dyslipidemia seen between 2008 and 2009 was conducted.

RESULTS: A total of 1061 patients from across Canada were reviewed. The mean age was 57 years, $61 \%$ were male, $75 \%$ were Caucasian, $39 \%$ were hypertensive and $29 \%$ had a smoking history. The Framingham risk score (FRS) was used by $61 \%$ of physicians for CVD risk assessment.

ssessment of individual cardiovascular disease (CVD) risk is an Aessential component of preventive cardiology. In North America, the Framingham risk score (FRS) has been most widely utilized in primary care, and represents a well-validated risk assessment tool (1). Use of the FRS for CVD risk stratification was endorsed in the 2006 Canadian Cardiovascular Society (CCS) position statement on the diagnosis and treatment of dyslipidemias (2), and use of the modified FRS for total CVD risk was first endorsed in the 2009 Canadian lipid guidelines (3).

Despite guideline recommendations, CVD risk assessment by primary care physicians remains highly variable (4). In a recent survey of 846 Canadian physicians, there were significant gaps in both the choice of risk assessment tools used in primary care, and the knowledge regarding novel and emerging factors of risk (5). In an attempt to further characterize this issue, we conducted a retrospective chart audit of Canadian primary care physicians to identify knowledge and action gaps with respect to risk assessment and lipid treatment.

\section{METHODS}

A total of 105 Canadian primary care physicians were invited to participate as part of the Primary Care Audit of Global Risk Management (PARADIGM) registry. These physicians were identified to provide balanced national representation from nine of the 10 Canadian provinces. Using a structured CRF, physicians were asked to provide information on risk assessment and treatment via a retrospective chart audit of 10 patients within their practice seen within the preceding calendar year (2008 to 2009).

Eligible patients included statin-naive asymptomatic males $>40$ years of age or females $>50$ years of age assessed within the past 12 months, at which time cardiovascular risk stratification was
Overall, $48 \%$ of patients were considered to be low risk, $40 \%$ intermediate and $12 \%$ high risk by physician assessment. This was a significant overestimation of risk $(\mathrm{P}<0.0001)$ compared with centrally derived FRS of $64 \%$, $26 \%$ and $10 \%$, respectively. Risk was overestimated more often in women $(\mathrm{P}<0.002)$. Statin therapy was prescribed to 390 patients $(37 \%)$; however, $36.5 \%$ of patients who were eligible for treatment, according to national guidelines, were not treated, while $19.9 \%$ of noneligible patients did receive therapy.

CONCLUSIONS: Despite guideline recommendations, the FRS was underutilized by Canadian primary care physicians. There was considerable discrepancy between centrally derived and physician-derived risk scores. Appropriate statin therapy appeared to be underprescribed by physicians despite an overestimation of risk. Improved dissemination of risk stratification tools and guideline recommendations are needed to optimize CVD risk reduction in primary care.

Key Words: Cardiovascular disease; Lipids; Primary prevention; Risk stratification; Treatment gap

performed along with measurement of fasting glucose and lipid levels. Patients were excluded if they had a history of diabetes or documented atherosclerosis (myocardial infarction, stroke, peripheral arterial disease, angina, revascularization, angiographic coronary artery disease, transient ischemic attack or $>50 \%$ carotid stenosis). Patients receiving any lipid-lowering therapy, including statins, were also excluded.

Patient demographics, risk factors, physical measures, laboratory results including glucose and lipid profile, and concomitant medications were abstracted. In addition, physicians were asked to estimate their patient's CVD risk as low, intermediate or high. CRFs were faxed to the Canadian Cardiovascular Research Network (CCRN) coordinating centre for data analyses and interpretation. The present study was approved by a central ethics committee (IRB Services, Aurora, Ontario).

Continuous variables are expressed as mean \pm SD and categorical variables as proportions. Continuous variables in the two groups were compared using the Student's $t$ test; where applicable, $\mathrm{P}<0.05$ was considered to be statistically significant. Percentages were calculated on the basis of total responses.
Overall physician and patient cohort
Of the 95 participating physicians, $85 \%$ were men, with a median age of between 50 and 60 years $(44 \%)$ and median years in practice $>20$ (73\%). The majority $(76 \%)$ attended $>5$ continuing medical educa- tion programs per year. Solo practitioners comprised $51 \%$ of the group, with $75 \%$ practicing in an urban setting and $20 \%$ having an academic affiliation.
The audit included 1061 patients (75\% Caucasian) from all Canadian provinces excluding Alberta. Ontario was represented by

${ }^{1}$ Canadian Cardiovascular Research Network, Brampton; ${ }^{2}$ Department of Medicine, McMaster University, Hamilton; ${ }^{3}$ Division of Cardiac Surgery,

Keenan Research Centre in the Li Ka Shing Knowledge Institute of St Michael's Hospital, University of Toronto, Toronto, Ontario; ${ }^{4}$ Division of

Cardiology, Medical College of Georgia at Augusta University, Augusta, Georgia, USA

Correspondence: Dr Milan Gupta, Canadian Cardiovascular Research Network, 3 Conestoga Drive, Suite\# 301, Brampton, Ontario L6Z 4 N5.

Telephone 905-453-8885, fax 905-456-8885, e-mail mkgupta@rogers.com 
TABLE 1

General characteristics of the cohort and according to sex

\begin{tabular}{lcccc}
\hline Characteristic & $\begin{array}{c}\text { Overall } \\
(\mathbf{n}=\mathbf{1 0 6 1 )}\end{array}$ & $\begin{array}{c}\text { Male } \\
(\mathbf{n}=\mathbf{6 4 9 )}\end{array}$ & $\begin{array}{c}\text { Female } \\
(\mathbf{n}=\mathbf{4 1 2})\end{array}$ & $\mathbf{P}$ \\
\hline Age, years & $57 \pm 9$ & $54 \pm 9$ & $60 \pm 9$ & $<0.01$ \\
$\begin{array}{l}\text { Current or past } \\
\text { smokers, \% }\end{array}$ & 29 & 33 & 23 & $<0.01$ \\
$\begin{array}{l}\text { Family history of } \\
\text { Panty }\end{array}$ & 20 & 22 & 18 & 0.0009
\end{tabular}

premature vascular

disease in a first-

degree relative, $\%$

$\begin{array}{lcccc}\text { Hypertension, \% } & 39 & 38 & 41 & <0.001 \\ \text { Blood pressure, mmHg } & 129 / 80 \pm 15 / 9 & 129 / 81 \pm 15 / 9 & 129 / 79 \pm 16 / 9 & 0.0002 \\ \text { BMl, kg/m² } & 28.7 \pm 5.6 & 29.0 \pm 4.8 & 28.0 \pm 6.8 & <0.01 \\ \text { Waist circumference, } & 97 \pm 14 & 100 \pm 12 & 91 \pm 16 & <0.01 \\ \mathrm{~cm} & & & & \end{array}$

Data presented as mean $\pm S D$ unless otherwise indicated. BMI Body mass index

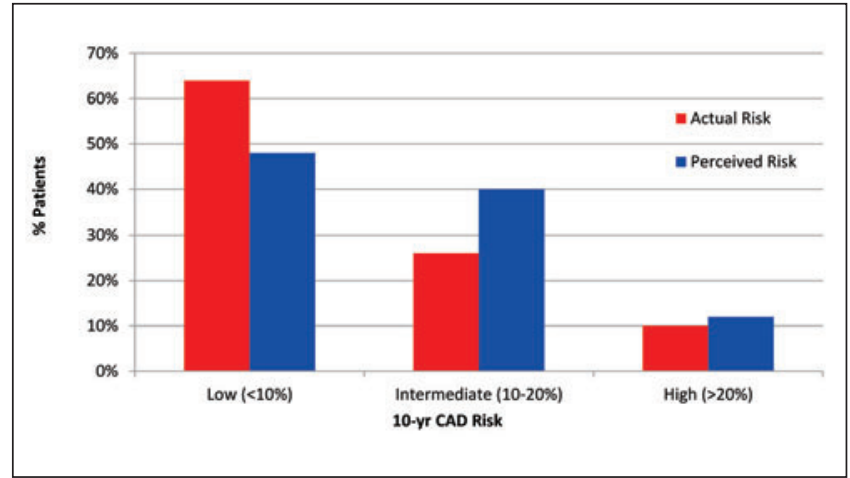

Figure 1) Framingham risk score. Actual (centrally calculated) versus perceived (physician evaluation) risk score. CAD Coroanary artery disease; yr Year

$36 \%$ of patients, followed by British Columbia (24\%) and Manitoba (16\%), with the remaining provinces comprising $24 \%$ of the cohort. The general characteristics of the cohort and according to sex are summarized in Table 1 . The mean $( \pm \mathrm{SD})$ age was $57 \pm 9$ years and $61.2 \%$ were male. Hypertension was identified in $39 \%$ of patients, and $29 \%$ were current or past smokers. One-fifth (20\%) of patients reported having a family history of premature CVD. Anthropometric indexes in the cohort included a mean body mass index (BMI) of $28.7 \pm 5.6 \mathrm{~kg} / \mathrm{m}^{2}$ and waist circumference of $97 \pm 14 \mathrm{~cm}$. However, BMI was documented in only $54 \%$ of charts and waist circumference in only $23 \%$ of charts. Measurement of high-sensitivity C-reactive protein (hs-CRP) levels were documented in only $7 \%$ of patients. Medications of relevance included acetylsalicylic acid in $20 \%$ of patients, angiotensin-converting enzyme inhibitors/angiotensin receptor blockers in $26 \%$, diuretics in $19 \%$, calcium channel blockers in $7 \%$ and beta blockers in $5 \%$.

Physicians were asked to indicate their usual method of CVD risk stratification. The FRS was the primary method of risk determination by $61 \%$ of physicians. Other options for risk assessment included overall clinical judgment (22\%), counting the number of risk factors $(13 \%)$, use of other validated risk scores $(3 \%)$ and measurement of hs-CRP levels (1\%).

Overall, physicians classified $48 \%$ of patients as low risk, $40 \%$ at intermediate risk and $12 \%$ at high risk for future cardiovascular events. To evaluate the concordance between physician-reported assessment of risk and actual risk, FRS was calculated centrally for each patient and compared with physician-reported risk. As noted in Figure 1, centrally derived risk calculation classified $64 \%$ of individuals as low risk, $26 \%$ as intermediate risk and $10 \%$ as high risk. This comparison revealed that physician assessment led to a significant underestimation
TABLE 2

Main laboratory parameters for the overall cohort and according to sex

\begin{tabular}{lcccc}
\hline $\begin{array}{l}\text { Baseline } \\
\text { parameter }\end{array}$ & Overall & Male & Female & P \\
\hline $\begin{array}{l}\text { Total cholesterol, } \\
\mathrm{mmol} / \mathrm{L}\end{array}$ & $5.9 \pm 1.0$ & $5.8 \pm 1.0$ & $6.1 \pm 1.0$ & $<0.0001$ \\
$\mathrm{HDL}, \mathrm{mmol} / \mathrm{L}$ & $1.3 \pm 0.4$ & $1.2 \pm 0.3$ & $1.5 \pm 0.4$ & $<0.0001$ \\
$\mathrm{LDL}, \mathrm{mmol} / \mathrm{L}$ & $3.8 \pm 0.9$ & $3.7 \pm 0.9$ & $3.9 \pm 0.9$ & 0.0009 \\
$\mathrm{TG}, \mathrm{mmol} / \mathrm{L}$ & $1.8 \pm 1.2$ & $1.9 \pm 1.3$ & $1.6 \pm 0.8$ & $<0.001$ \\
Fasting blood & $5.4 \pm 0.8$ & $5.5 \pm 0.9$ & $5.3 \pm 0.6$ & 0.0002 \\
$\quad$ glucose, $\mathrm{mmol} / \mathrm{L}$ & & & & \\
A1C, \%* & $5.7 \pm 0.7$ & $5.7 \pm 0.8$ & $5.7 \pm 0.4$ & 1.0 \\
hsCRP, $\mathrm{mg} / \mathrm{L}^{\dagger}$ & $3.8 \pm 7.7$ & $3.6 \pm 7.5$ & $4.8 \pm 9.2$ & 0.02 \\
Creatinine, $\mu \mathrm{mol} / \mathrm{L}$ & $82 \pm 17$ & $88 \pm 15$ & $71 \pm 14$ & $<0.0001$ \\
\hline
\end{tabular}

${ }^{*} n=212 ;{ }^{\dagger} n=73$. A1C Glycated hemoglobin; HDL high-density lipoprotein; hsCRP High-sensitivity C-reactive protein; LDL Low-density lipoprotein; TG Triglycerides

of low-risk patients $(\mathrm{P}<0.0001)$ and overestimation of intermediaterisk patients $(\mathrm{P}<0.0001)$.

\section{Sex differences}

There were 649 men (61\%) and 412 women in the present study. Significant sex differences were identified in this cohort (Table 1). Compared with women, men were younger based on the inclusion criteria $(54 \pm 9$ years versus $60 \pm 8$ years; $\mathrm{P}<0.01)$, had higher BMI $(29 \pm 5$ $\mathrm{kg} / \mathrm{m}^{2}$ versus $\left.28 \pm 7 ; \mathrm{P}<0.01\right)$, and greater waist circumference $100 \pm 12$ $\mathrm{cm}$ versus $91 \pm 16 \mathrm{~cm} ; \mathrm{P}<0.01)$. No significant differences in blood pressure $(129 / 81 \pm 15 / 9 \mathrm{mmHg}$ versus $129 / 79 \pm 16 / 9 \mathrm{mmHg})$ or family history of CVD (22\% versus $18 \%$ ) were noted. Men were more often smokers $(33 \%$ versus $18 \%$; $<0.01)$. In both men and women, physician-reported CVD risk varied significantly from the centrally derived FRS risk score. In men, low risk was underestimated (40\% versus $48 \%$; $\mathrm{P}<0.04)$ and intermediate risk was overestimated (45\% versus $39 \%$; $\mathrm{P}<0.03)$. Perceived FRS was concordant $(15 \%$ by both scores) for high-risk males.

More striking differences in risk assessment were apparent in women. Notably, low risk was underestimated (60\% versus $93 \%$; $\mathrm{P}<0.0001$ ), intermediate risk was overestimated ( $6 \%$ versus versus $33 \% ; \mathrm{P}<0.0001)$ and high risk was also overestimated $(1 \%$ versus $7 \%$; $\mathrm{P}<0.002$ ).

Based on the centrally derived scores, more women compared with men had low FRS (93\% versus 46\%; $\mathrm{P}<0.0001$ ) and fewer women had intermediate FRS (6\% versus 39\%; $\mathrm{P}<0.0001$ ). Consequently, fewer women compared with men had high FRS ( $1 \%$ versus $15 \%$; $\mathrm{P}<0.0001)$.

\section{Lipid and other laboratory parameters}

Table 2 summarizes the main laboratory parameters for the overall cohort and according to sex. The mean total cholesterol level was $5.9 \pm 1.0 \mathrm{mmol} / \mathrm{L}$, low-density lipoprotein cholesterol (LDL-C) was 3.8 $\pm 0.9 \mathrm{mmol} / \mathrm{L}$ and the high-density lipoprotein cholesterol (HDL-C) was $1.3 \mathrm{mmol} / \mathrm{L}$. The mean fasting glucose level was $5.4 \pm 0.8 \mathrm{mmol} / \mathrm{L}$ and hemoglobin A1c was $5.7 \pm 0.7 \%$. The mean serum creatinine level was $82 \pm 17 \mathrm{umol} / \mathrm{L}$. hs-CRP was documented in $7 \%$ of cases, with a mean value of $3.8 \mathrm{mg} / \mathrm{L}$.

\section{Pharmacological treatment}

Based on the risk assessment performed at the time of the physician visit, statin therapy was prescribed in 390 (36.8\%) of the overall population. Specifically, statin prescription was observed in 9\%, 55\% and $90 \%$ of low-, intermediate- and high-risk individuals, respectively, based on physician-derived risk estimation (Figure 2). Based on centrally derived FRS, 411 patients should have been prescribed a statin according to national lipid guidelines. 


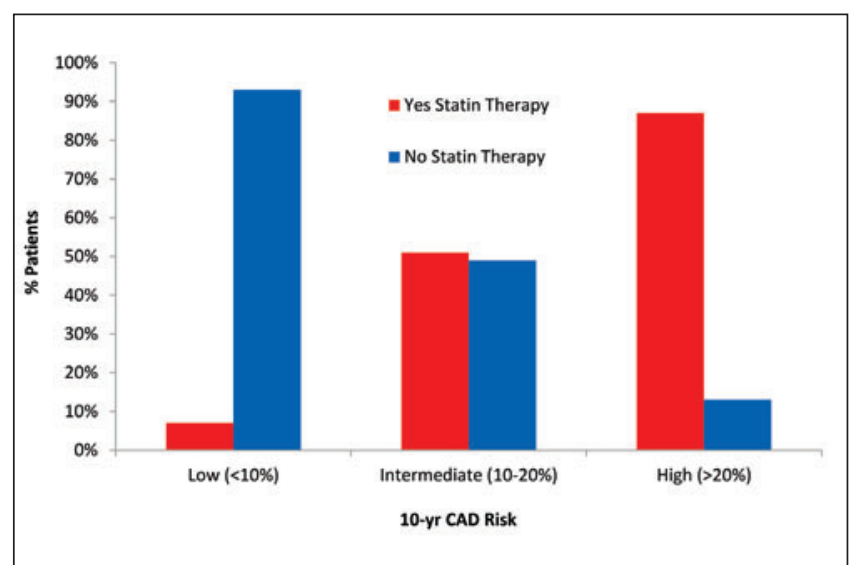

Figure 2) Statin treatment based on perceived (physician evaluation) Framingham risk score. CAD Coronary artery disease; yr Year

The mean LDL-C for patients prescribed statin was $4.4 \pm 1.0$, $4.2 \pm 0.8,4.3 \pm 1.0 \mathrm{mmol} / \mathrm{L}$ in the low-, intermediate- and high-risk categories respectively, as determined by physicians. The most commonly prescribed statin was rosuvastatin (63\%, mean dose $10.1 \mathrm{mg})$, followed by atorvastatin in $29 \%$ (mean dose $16.4 \mathrm{mg}$ ) and simvastatin in $7 \%$ (mean dose $24.5 \mathrm{mg}$ ). Non-statin therapies including niacin, fenofibrate, ezetimibe, gemfibrozil and cholestyramine were used in very few patients $(2.7 \%$ collectively). Combination therapy was also rarely used in this primary prevention population (2.4\%).

Subsequently assessed was whether statin prescription was appropriate based on risk level and baseline LDL-cholesterol level. There were 642 patients in whom the physician reported calculating the FRS (as opposed to other methods of CVD risk assessment), of which 232 should have been treated based on the 2006 CCS lipid guidelines. Central assessment of risk identified a total of 19 individuals at low risk, with an LDL-C $>5.0 \mathrm{mmol} / \mathrm{L}$, who according to guidelines, would be deemed eligible for statin therapy. Compared with this value, the actual number of patients treated with statin was nine. Similarly, the number of patients with a centrally calculated FRS in the intermediate risk category with an $\mathrm{LDL}>3.5 \mathrm{mmol} / \mathrm{L}$ and, hence, eligible for statin therapy was 147 , compared with 101 who were actually treated. In high-risk patients, 66 were eligible for treatment based on central assessment; however, the actual number treated was 57 . These results suggest consistent undertreatment $(72 \%)$ in all risk categories. At the same time, the analysis revealed that of the 410 patients who should not have been recommended statin therapy, 18 (5.1\%) in the low-risk group, $11(18.3 \%)$ in the intermediate-risk group and none in the high-risk group were treated (Figure 3A).

In the 419 patients in whom FRS was not calculated locally, there were 215 who should have been treated, 10 in the low risk, 147 in the intermediate risk and 58 in the high-risk group. Treatment occurred in four patients in the low-risk group, 97 in the intermediate-risk and 54 in the high-risk. Again, this indicates undertreatment $(72.1 \%)$ in individuals in whom FRS was not calculated. At the same time, the analysis revealed that of the 204 patients who should not have been on statin therapy in this subset, $13(7.6 \%)$ in the low-risk group, 26 $(36.1 \%)$ in the intermediate-risk group and none in the high-risk group were treated (Figure 3B)

In men, statins were prescribed to $9 \%, 54 \%$ and $89 \%$ of low-, intermediate- and high-risk patients, respectively. Similar percentages were observed in women: $8 \%, 57 \%$ and $93 \%$, respectively.

\section{DISCUSSION}

The primary objective of the PARADIGM retrospective audit was to determine how primary care physicians in Canada perform CVD risk assessment, and to evaluate the concordance between their assessment of risk and a centrally calculated FRS. A secondary objective was to
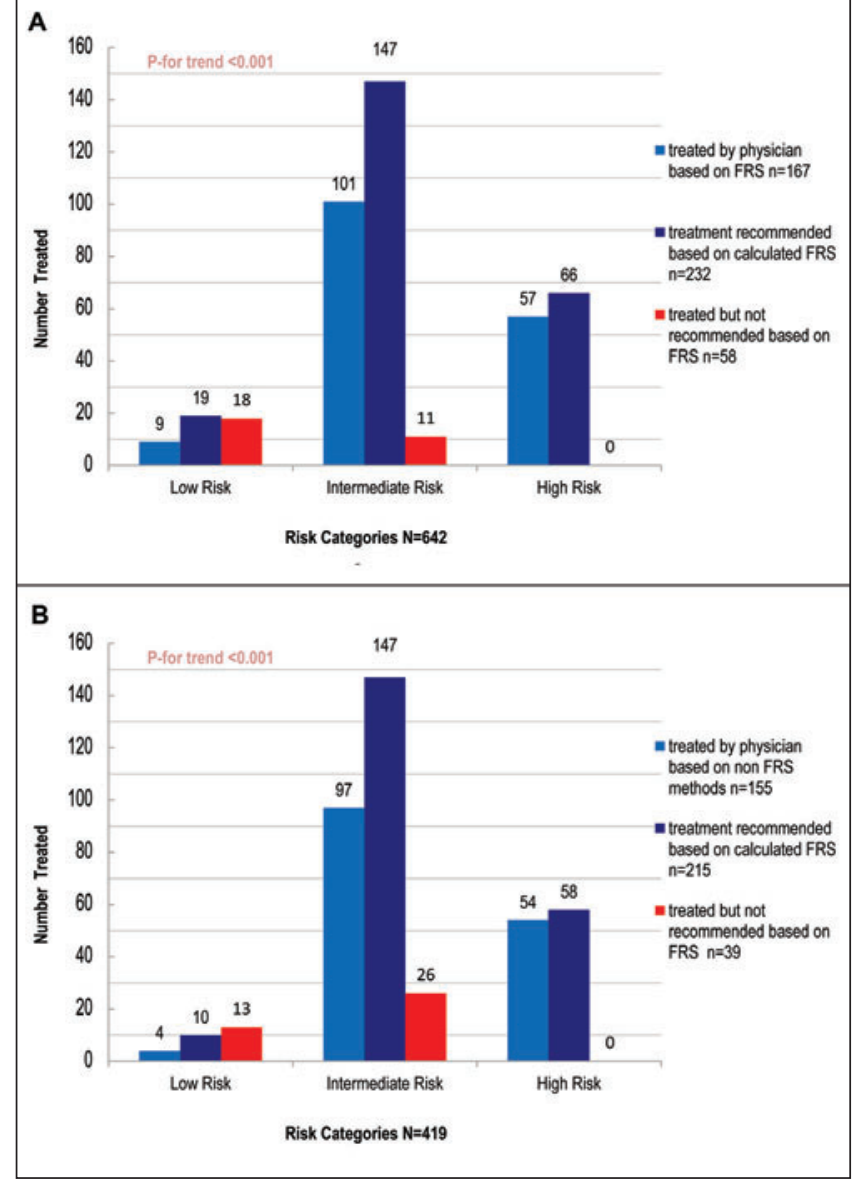

Figure 3) Actual statin treatment based on physician use of the Framingham risk score (FRS) (A) versus non-FRS risk assessment (B). This is compared with predicted treatment based on centrally calculated FRS and also shows treated patients who would likely not be eligible for treatment based on centrally calculated FRS

evaluate whether lipid-lowering therapy was prescribed appropriately based on physician-reported risk level.

Several important observations merit discussion. Only 64\% of primary care physicians reported using the FRS for global risk assessment. This finding is consistent with an earlier survey of 846 primary care physicians, in which $69 \%$ reported using FRS to assess risk (5). Although the limitations of the FRS are well characterized (6), the FRS remains the most widely recommended and validated risk calculator in North America. Despite Canadian guidelines recommending the use of the FRS, it is unclear why more than one-third of primary care physicians in PARADIGM chose to use other forms of risk assessment $(7,8)$.

Considerable discrepancy was noted between physician-reported risk and centrally calculated FRS. It is not clear why this difference exists unless physicians are imputing data that are different from what was abstracted from the chart. The implications of this are substantial in that only $72 \%$ of patients who should have been prescribed statin therapy were initiated while $7.1 \%$ of patients who likely should not have been treated were also initiated. It is notable that under prescribing occurred just as often in patients in whom the FRS was not used to risk stratify.

This also raises a broader issue of why national guidelines are not fully adopted. Recently, following an abstraction review of 53 American College of Cardiology/American Heart Association practice guidelines on 22 topics between 1984 and 2008, Tricoci et al (9) determined that there has been a $48 \%$ increase in the number of recommendations, with the largest increase occurring among class 
II recommendations, which are often perceived to be weak recommendations. Notably, these authors also reported that among the 16 current guidelines reporting levels of evidence, 1246 of the 2711 recommendations were based on expert opinion (level of evidence C) rather than on randomized controlled clinical trials. Guidelines have been perceived to be too long, complex and containing recommendations based on relatively weak evidence may contribute to clinical care gaps (10).

There remains considerable uncertainty regarding the thresholds for treatment in subjects at intermediate risk. In such patients, approximately $50 \%$ received statin therapy. In intermediate-risk patients in whom statin therapy was not used, the LDL level straddles the guideline treatment threshold of $3.5 \mathrm{mmol} / \mathrm{L}$. Because the Justification for the Use of Statins in Prevention: an Intervention Trial Evaluating Rosuvastatin (JUPITER) study findings (11) had not yet been incorporated into the guidelines, the use of hs-CRP was low and may have contributed to the uncertainty of treatment need. Multiple studies and various national guidelines now support the use of hs-CRP in such populations (12) for refinement of risk stratification. Other markers, such as Lp (a), HDL and LDL subtyping, and microalbuminuria have also been suggested as possible risk refinement tools for intermediate-risk patients. Imaging modalities, such as calcium score, B-mode carotid ultrasound and ankle-brachial index, have also been suggested as useful prognostic tools for risk stratification (13-15).

Our study demonstrates that primary care physicians tend to underestimate CAD risk in men more often than women. This is in contrast to previous studies suggesting a sex bias toward under recognition and undertreatment for women (16). The 2011 AHA/ACC update raised concerns about using FRS to estimate risk in women because it is difficult for women $<75$ years of age to exceed a ' $10 \%$ in 10 year' risk threshold. Lifetime risk may be a better tool for women and was incorporated into the 2013 AHA/ACC guidelines on blood cholesterol (14). Such commentary may be a possible explanation for why primary care physicians significantly overestimated risk in women.

Also of concern has been the role of statin therapy for primary prevention in women. A previous meta-analysis failed to show a benefit (17), although the recent JUPITER trial did demonstrate a small absolute benefit but the number needed to treat was greater than in men. Benefit has since been further confirmed by the Cholesterol Treatment Trialists Collaboration (18); however, such knowledge was not readily available at the time of the present audit.

\section{REFERENCES}

1. Eichler K, Puhan MA, Steurer J, Bachmann LM. Prediction of first coronary events with the Framingham risk score: A systematic review. Am Heart J 2007;153:722-31.

2. McPherson R, Frohlich J, Fodor G, Genest J; Canadian Cardiovascular Society. Canadian Cardiovascular Society position statement recommendations for the diagnosis and treatment of dyslipidemia and prevention of cardiovascular disease. Can J Cardiol 2006;22:913-27.

3. Genest J, McPherson R, Frohlich J, et al. 2009 Canadian Cardiovascular Society/Canadian guidelines for the diagnosis and treatment of dyslipidemia and prevention of cardiovascular disease in the adult - 2009 recommendations. Can J Cardiol 2009;25:567-79.

4. Stone JA. How can we know what doctors know? Can J Cardiol 2011;28:11-3.

5. Gupta M, Singh N, Tsigoulis M, et al. Perceptions of Canadian primary care physicians towards cardiovascular risk assessment and lipid management. Can J Cardiol 2012;28:14-9.

6. Grundy SM, Cleeman JI, Merz CN, et al. Implications of recent clinical trials for the National Cholesterol Education Program Adult Treatment Panel III guidelines. Circulation 2004;110:227-39.

7. Shaneyfelt TM, Centor RM. Reassessment of clinical practice guidelines: Go gently into that good night. JAMA 2009;301:868-9.

8. Cabana MD, Rand CS, Powe NR, et al. Why don't physicians follow clinical practice guidelines? A framework for improvement. JAMA 1999;282:1458-65.
Inappropriate or inadequate risk stratification can result in inappropriate or inadequate treatment. Although our study involved seasoned physicians, we demonstrated that $36.5 \%$ of patients who met treatment guidelines were not treated due to misclassification. In contrast, $19.9 \%$ of misclassified patients who likely should not have been treated were treated. Consequently, it is important to develop better risk stratification tools that will be more widely and successfully adopted by primary care physicians.

It is of interest that physicians overestimated risk but undertreated based on the risk assessment. The decision to initiate statin therapy is dependent on more than a mere risk score (14). Patient preferences, potential side effects, comorbidities, concomitant drugs, family history and nonincorporated risk factors all play a role (19). One of the limitations of our study was the inability to determine how much these factors played into physician decision making. Another limitation was the short duration of analysis. Some patients may not have initially been recommended statin therapy to allow an adequate attempt at dietary modification and lifestyle changes. Others may have started treatment but stopped shortly after (20). Our study also had some selection bias in that the participating physicians likely had more of an interest in lipid management and, thus, may not be fully representative of the broader primary care physician population.

Since the completion of the present retrospective audit, the national lipid guidelines have undergone two further guidances $(21,22)$. In addition, in 2013, the AHA/ACC challenged the notion of treatment targets and introduced a new risk calculator to the public domain. The impact of conflicting guidelines and conflicting guidance will potentially make it even more difficult for primary care physicians to appropriately risk stratify their patients.

FUNDING: Supported, in part, by an investigator-initiated grant from Astra Zeneca Canada to Canadian Cardiovascular Research Network.

DISCLOSURES: Milan Gupta has received speaking honoraria, consulting fees, and/or research grants from AstraZeneca, Merck, and Pfizer. Michelle Tsigoulis and Mahesh Kajilhave have no financial disclosures or conflicts of interest to declare. Subodh Verma has received speaking honoraria, consulting fees, and/or research grants from Abbott, AstraZeneca, Boehringer Ingelheim, Merck/Schering-Plough, Novartis, Pfizer and sanofi-aventis. Narendra Singh has received speaking honoraria, consulting fees and/or research grants from AstraZeneca, Merck and Pfizer.

9. Tricoci P, Allen JM, Kramer JM, Califf RM, Smith SC Jr. Scientific evidence underlying the ACC/AHA clinical practice guidelines. JAMA 2009; 301:831-41

10. Institute of Medicine (U.S.) Committee on Standards for Developing Trustworthy Clinical Practice Guidelines. Clinical Practice Guidelines We Can Trust. Washington, DC: National Academics Press; 2011.

11. Ridker PM, Danielson E, Fonseca FA, et al. Rosuvastatin to prevent vascular events in men and women with elevated C-reactive protein. N Engl J Med 2008; 359:2195-207.

12. Buckley DI, Fu R, Freeman M, et al. C-reactive protein as a risk factor for coronary heart disease: A systematic review and metaanalyses for the U.S. Preventative Services Task Force. Ann Intern Med 2009;151:483-95.

13. Polak JF, Pencina MJ, Pencina KM, O'Donnell CJ, Wolf PA, D'Agostino RB Sr. Carotid-wall intima-media thickness and cardiovascular events. N Eng J Med 2011;365:213-21.

14. Stone NJ, Robinson JG, Lichtenstein AH, et al. 2013 ACC/AHA guideline on the treatment of blood cholesterol to reduce atherosclerotic cardiovascular risk in adults; a report of the American College of Cardiology/American Heart Association Task Force on Practice Guidelines. J Am Coll Cardiol 2014;63:2889-934.

15. Knapper JT, Blaha M, Berman D, et al. Effectiveness of coronary artery calcium scoring for long term risk stratification in patients with or without a family history of coronary heart disease. J Am Coll Cardiol 2014;63:a1269. 
16. Ridker PM, Buring JE, Rifai N, Cook NR. Development and validation of improved algorithms for the assessment of global cardiovascular risk in women: The Reynolds Risk Score. JAMA 2007;297:611-9.

17. Brugts JJ, Yetgin T, Hoeks SE, et al. The benefits of statins in people without established cardiovascular disease but with cardiovascular risk factors: meta-analysis of randomised controlled trials. BMJ 2009;338:b2376.

18. Cholesterol Treatment Trialists' (CTT) Collaboration. Efficacy and safety of LDL-lowering therapy among men and women: Meta-analysis of individual data from 174,000 participants in 27 randomized trials. Lancet 2015;385:1397-405.

19. Mancini GB, Baker S, Bergeron J, et al. Diagnosis, prevention, and management of statin adverse effects and intolerance: Proceedings of a Canadian Working Group Concensus Conference. Can J Cardiol 2011;27:635-62
20. Jackevicius CA, Mamdani M, Tu JV. Adherence with statin therapy in elderly patients with and without acute coronary syndromes JAMA 2002;288:462-7.

21. Anderson TJ, Gregoire J, Hegele RA, et al. 2012 update of the Canadian Cardiovascular Society guidelines for the diagnosis and treatment of dyslipidemia for the prevention of cardiovascular disease in the adult. Can J Cardiol 2013;29:151-67.

22. Anderson TJ, Gregoire J, Hegele RA, et al. Are the ACC/AHA Guidelines on the treatment of blood cholesterol a game changer? A perspective from the Canadian Cardiovascular Society dyslipidemia panel. Can J Cardiol 2014;30:377-80. 